\title{
Performance Enhancement of Achievable Throughput in Multi-Taper Spectrum Sensing
}

\author{
Mohamed Ismail \\ Electronics \& Communications \\ Department \\ Modern Academy for Engineering and \\ Technology \\ Cairo, Egypt
}

\author{
Atef Ghuniem \\ Department of Electrical Engineering \\ Suez Canal University \\ Ismailia, Egypt
}

\author{
Abdelhamid Gaafar \\ Electronics \& Communications \\ Department \\ ASST- Arab Academy for Science, \\ Technology \& Maritime Transport \\ Cairo, Egypt
}

\begin{abstract}
Nowadays, many researchers pay much attention to spectrum sensing techniques. Many methods are proposed earlier to sense the spectrum of different selective bands. In this paper, a proposed Multi-Taper Spectrum Sensing (MTSS) technique is used to sense the spectrum trying to enhance the achievable throughput criteria in a small sensing time. The simulation results of the proposed MTSS will be compared to the results of Energy Detection Spectrum Sensing (EDSS) method for two scenarios. The first scenario is the single user detection while the second one is the Co-operative spectrum sensing detection.
\end{abstract}

Keywords-Multi-Taper Spectrum Sensing; Energy Detection; Cooperative Spectrum Sensing; Throughput;

\section{INTRODUCTION}

Cognitive Radio Spectrum Sensing (CRSS) is considered a hot spot for many researchers. They used CRSS to efficiently utilize the overall band. This can be done by exploiting the free frequency bands (spectrum holes) [1]. Hence, this frequency band can be utilized by SUs in the current time slot. There are many spectrum sensing techniques among are used earlier. The most used technique is EDSS, because it does not require prior information about the Primary User (PU) signaling and has minimal complexity. Such advantages come at the expense of moderate performance due to the use of single rectangular window. There are many problems in spectrum sensing such as hidden node and trade-off between time of sensing and transmission time.

A survey is done and relevant studies proposed earlier are presented in this section. The first study [2] discussed the depleted frequency spectrum in wireless communication systems. Authors of [3] used The Energy Detector (ED) to detect an unknown signal in the presence of additive noise. The signal is detected by emulating the output of the energy detector with a threshold which relies on the noise floor [4].Thomson [5], works slightly like but it utilizes various orthonormal prototype filters to get better the variance of estimated power and minimize the leakage. Multi-Taper spectrum sensing (MTSS) [6], utilizes orthogonal tapers; known as the Discrete Prolate Spheroidal Sequence (DPSS). It results a single spectrum estimate with minimal spectral leakage and good variance. Authors of [7] derived that MTSS is an approach of the optimal spectrum estimate (the Maximum-likelihood method) with minimal computation complexity. Authors of [8] enhanced the achievable throughput using co-operative spectrum sensing.
Cognitive radios can operate in both authorized and unauthorized bands. Users with a license is operate in a specific band are called primary users (PU's) and have priority access to that band. Cognitive radio users/secondary users (SU's) are capable of sensing the radio environment and determining spectrum holes. SU's can make use of the best available spectrum holes in a pragmatic manner, as long as they do not overlap with the authorized users. In this manner, cognitive radios can overcome the problem of spectrum reduction and underutilization [8].

In this paper, MTSS is used to maximize the SU throughput and decreasing the time of sensing more than EDSS. Detection probability vs. false alarm probability and throughput vs. sensing time are simulated at different scenarios of EDSS and MTSS (either single user detection or multiple user detection); trying to have a higher achievable throughput of the proposed co-operative MTSS than conventional co-operative EDSS in small sensing time.

The rest of the paper is organized as follows, In Section II the proposed primary user system model, the effect of MTSS on the detection probability and the false alarm probability are presented. Sensing throughput is mentioned in Section III. In Section IV Cooperative spectrum sensing is presented. Discussion of the numerical results, simulations in the mentioned MTSS Cooperative spectrum sensing and Conventional Cooperative spectrum sensing are presented in Section V. Finally, in Section VI the conclusions are given.

\section{SYSTEM MODEL}

The proposed system model is consisted of OFDM signaling scheme for the PU as shown in Fig.1. The PU transmitter with N-subcarriers (N-IFFT/FFT) transmits OFDMQPSK signal over each subcarrier. The CR transceiver also consisting of (N-IFFT/FFT) processor which performs tasks of sensing and transmission as shown in the CR frame structure.CR frame structure is divided into two parts sensing times $(\mathrm{S})$ and transmission time (T-S) as the total frame time is T. Additionally Energy detector or Multi-taper spectrum detector is added.

Energy detector is a widespread way, also known as Periodogram or radiometry, is the most common approach of spectrum sensing because of its minimum computational and implementation complexities. The reliability of this way is reduced by: first, the estimated spectrum variance $\hat{s}(\mathrm{f})$ at every frequency ' $\mathrm{f}$ ' (the spectrum often appears noisy) while the 
second, is the leakage of power across different frequencies. The leakage is outcome due to the fact that we select a finite band of signal, which equivalent to multiplying the selected

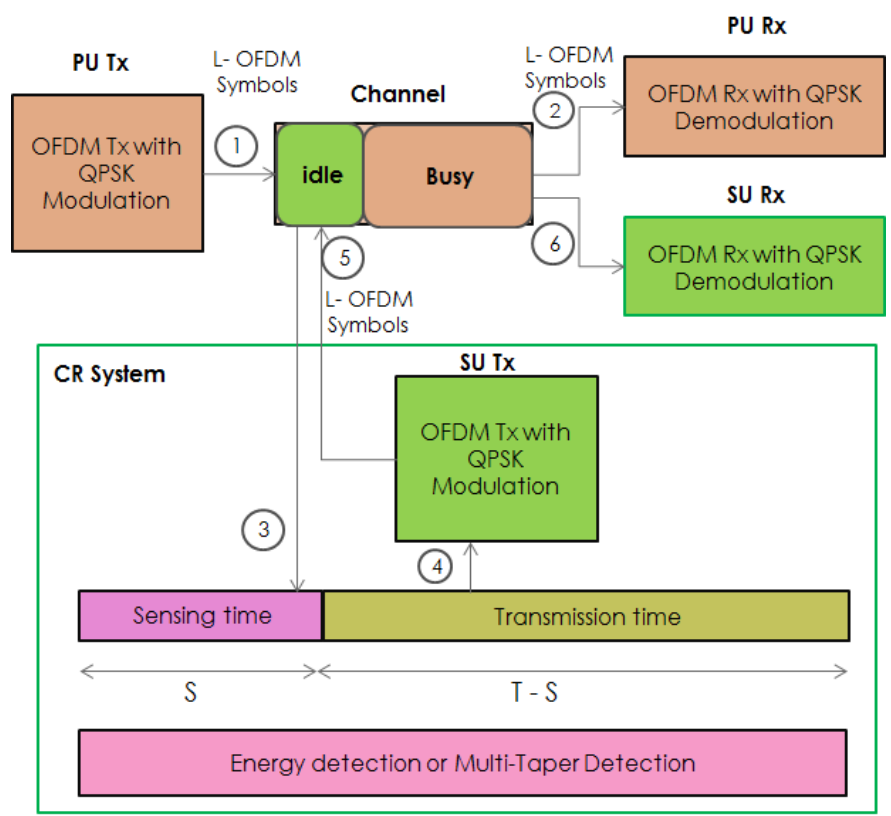

Fig.1. Proposed System Model Structure

band of signal by a rectangular window. The Fourier transform of a rectangular taper (window) produces multiple side bands and this causes the leakage of power problem as shown in Fig.2. A solution to minimize the leakage in the frequency domain is to first multiply the signal in the time domain with a non-rectangular window described by a Fourier transform with less energy in its side bands as shown in Fig.3 [9]. Of course, the usage of a window which also called a taper affects the estimate by minimizing leakage but it does not change the variance of the estimate at every frequency f. A common approach to minimize the variance is through using multi-taper spectrum estimation and taking the average between them.

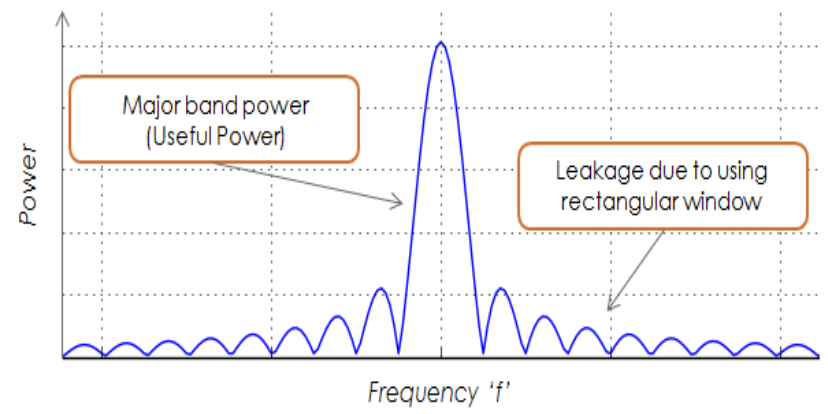

Fig.2. Leakage of power problem



Fig.3. The used K-tapers with low spectral leakage side band power

The difference between the estimated spectrum of multitaper spectrum estimation and conventional estimator (Energy detector) is discussed in this section. Assuming that the received PU signal, at $\mathrm{CR}$ receiver, is sampled to generate a finite discrete time samples series $\left\{\mathrm{R}_{t} ; t=0,1,2, \ldots \mathrm{N}-1\right\}$, where $t$ is the time index. The discrete time samples are multiplied by various tapers $\lambda_{k}(\mathrm{~N}, \mathrm{~B})$ such that B is the selected bandwidth. The associated eigenvalues of the $k^{\text {th }}$ taper is $v_{k}(\mathrm{~N}, \mathrm{~B})$. The result of multiplication is applied to a Fourier Transform to compute the energy concentrated in the bandwidth $(-B, \mathrm{~B})$ cantered at frequency $\mathrm{f}$ [9]. The power spectral density estimation of MTSS detector is shown in (1) [7]:

$$
\mathrm{S}_{M T S S}\left(f_{i}\right)=\frac{\sum_{K=0}^{K-1}\left(\left(v_{k}(\mathrm{~N}, \mathrm{~B})\left|\sum_{t=0}^{N-1} R_{t} \cdot \lambda_{(t, k)}(\mathrm{N}, \mathrm{B}) e^{-j 2 \pi f_{i} t}\right|^{2}\right)\right.}{\sum_{K=0}^{K-1} v_{k}(\mathrm{~N}, \mathrm{~B})}
$$

Where $\mathrm{S}_{M T S S}\left(f_{i}\right)$ is the power spectrum density of multi-taper spectrum estimator. Otherwise the power spectrum density estimation of conventional system (Energy Detector) as shown at (2):

$$
\mathrm{S}_{E D}(f)=\frac{1}{N}\left|\sum_{l=0}^{N-1} R_{t} e^{-j 2 \pi f t}\right|^{2}
$$

To evaluate the MTSS performance, suppose that the probability of detection $P_{d}$, the false alarm probability $P_{f}$ and the miss detection probability $P_{m} . P_{d}$ is the probability that $\mathrm{CR}$ detector decides correctly the presence of the PU's signal, $P_{f}$ is the probability that $\mathrm{CR}$ detector decides the PU's signal is present when it is absent, and $P_{m}$ is the probability that CR miss to detect the PUs signal when it is present. The binary hypothesis test for CR spectrum sensing at the $l^{\text {th }}$ time is given by:

$$
\begin{aligned}
& H_{0}: \mathrm{R}_{t}(l)=n_{t}(l) \\
& H_{1}: \mathrm{R}_{t}(l)=s_{t}(l)+n_{t}(l)
\end{aligned}
$$

Where $H_{0}$ indicates PU absent, $H_{1}$ indicates PU present, $l=0,1,2 \ldots \mathrm{L}-1$ is the received samples length, $R_{t}(l)$ denotes CR received signal, $n_{t}(l)$ is noise and $s_{t}(l)$ is PU transmitted samples. The transmitted primary user samples is affected by the zero mean additive white Gaussian noise 
$n_{t}(l) \square C \mathrm{~N}\left(0, \sigma_{n}^{2}\right)$.We now consider the mean (E), and the variance $(\mathrm{VAR})$ of the decision statistic $\operatorname{DEC}_{M T S S}\left(\mathrm{f}_{i}\right)$ for both hypotheses. The decision statistics over L samples using MTSS detector is as follows [7]:

$$
\begin{gathered}
\mathrm{DEC}_{M T S S}\left(\mathrm{f}_{i}\right)=\sum_{l=0}^{L-1} \frac{\sum_{K=0}^{K-1}\left(v_{k}(\mathrm{~N}, \mathrm{~B})\left|\sum_{t=0}^{N-1} R_{t} \cdot \lambda_{(t, k)}(\mathrm{N}, \mathrm{B}) e^{-j 2 \pi f_{i} t}\right|^{2}\right)}{\sum_{K=0}^{K-1} v_{k}(\mathrm{~N}, \mathrm{~B})} \\
P_{f}^{M T S S}\left(\mathrm{f}_{i}\right)=\mathrm{P}\left(\mathrm{DEC}_{M T S S}\left(\mathrm{f}_{i}\right)>\eta / \mathrm{H} 0\right) \\
P_{f}^{M T S S}\left(\mathrm{f}_{i}\right)=Q\left(\frac{\eta-\mathrm{E}\left(\mathrm{DEC}_{M T S S}\left(\mathrm{f}_{i}\right) / \mathrm{H} 0\right)}{\sqrt{V A R\left(\mathrm{DEC}_{M T S S}\left(\mathrm{f}_{i}\right) / \mathrm{H} 0\right)}}\right)
\end{gathered}
$$

Where $\mathrm{Q}($.$) denotes the complementary Gaussian distribution$ function (CGDF) and $\eta$ represents the detection threshold.

$$
\begin{gathered}
P_{d}^{M T S S}\left(\mathrm{f}_{i}\right)=\mathrm{P}\left(\mathrm{DEC}_{M T S S}\left(\mathrm{f}_{i}\right)>\eta / \mathrm{H} 1\right) \\
P_{d}^{M T S S}\left(\mathrm{f}_{i}\right)=Q\left(\frac{\eta-\mathrm{E}\left(\mathrm{DEC}_{M T S S}\left(\mathrm{f}_{i}\right) / \mathrm{H} 1\right)}{\sqrt{V A R\left(\mathrm{DEC}_{M T S S}\left(\mathrm{f}_{i}\right) / \mathrm{H} 1\right)}}\right) \\
P_{m}^{M T S S}\left(\mathrm{f}_{i}\right)=\mathrm{P}\left(\mathrm{DEC}_{M T S S}\left(\mathrm{f}_{i}\right)<\eta / \mathrm{H} 1\right) \\
P_{m}^{M T S S}\left(\mathrm{f}_{i}\right)=1-Q\left(\frac{\eta-\mathrm{E}\left(\mathrm{DEC}_{M T S S}\left(\mathrm{f}_{i}\right) / \mathrm{H} 1\right)}{\sqrt{V A R\left(\mathrm{DEC}_{M T S S}\left(\mathrm{f}_{i}\right) / \mathrm{H} 1\right)}}\right)
\end{gathered}
$$

Where [7]

$$
\mathrm{E}\left(\mathrm{DEC}_{M T S S}\left(\mathrm{f}_{i}\right)>\eta / \mathrm{H} 0\right)=\mathrm{KL}
$$

$$
\begin{gathered}
\operatorname{VAR}\left(\operatorname{DEC}_{M T S S}\left(\mathrm{f}_{i}\right)>\eta / \mathrm{H} 0\right)=2 C^{2} L v_{\Sigma} \\
P_{f}^{M T S S}=Q\left(\frac{\eta-K L}{\sqrt{2 C^{2} L v_{\Sigma}}}\right)
\end{gathered}
$$

Where

$$
\begin{gathered}
C=\frac{1}{\sum_{k=0}^{K-1} v_{k}} \\
v_{\sum}=\left(\sum_{k=0}^{K-1} v_{k}\right)^{2}+2 v_{0} v_{1}+2 v_{0} v_{2}+\ldots . .+2 v_{0} v_{K-1} \\
+2 v_{1} v_{2}+2 v_{1} v_{3}+\ldots . .+2 v_{1} v_{K-1}+2 v_{2} v_{3} \\
+\ldots \ldots .2 v_{2} v_{K-1}+\ldots+2 v_{k-2} v_{K-1} \\
P_{d}^{M T S S}=Q\left(\frac{\eta-K L(1+\mathrm{SNR})}{\sqrt{\left.2 C^{2} L v_{\Sigma}+4 C^{2} L v_{\Sigma} \mathrm{SNR}\right)}}\right)
\end{gathered}
$$

Such that SNR is assumed to be the received primary user signal-to-noise ratio. Energy Detection performance metrics [8]

$$
P_{f}^{E D}=Q\left(\frac{\eta-L}{\sqrt{2 L}}\right)
$$

$$
P_{d}^{E D}=Q\left(\frac{\eta-L(1+\mathrm{SNR})}{\sqrt{2 L+4 L \mathrm{SNR})}}\right)
$$

\section{SENSING THROUGHPUT TRADEOFF}

IN THE PAST SECTION, THE RELATION BETWEEN DETECTION probability and false alarm probability has been determined for Energy Detection and Multi-taper detection. In this section, we study the fundamental tradeoff between sensing capability and achievable throughput of the SU. Using Multitaper scheme, we will obtain that there exists indeed the optimal sensing time which achieves the best trade-off compared to ED. The channel capacities $C_{0}$ and $C_{1}$ are considered the capacities when the PU is absent or present respectively [8].

$$
\begin{gathered}
C_{0}=\log _{2}\left(1+\mathrm{SNR}_{s}\right) \\
C_{1}=\log _{2}\left(1+\frac{\mathrm{SNR}_{s}}{1+\mathrm{SNR}}\right)
\end{gathered}
$$

Where $\mathrm{SNR}_{s}$ is the secondary user signal to noise ratio, $P\left(\mathrm{H}_{0}\right)$ is the probability that the channel is idle and $P\left(\mathrm{H}_{1}\right)$ is the probability that the channel is busy. The throughputs $R_{0}$ and $R_{1}$ are considered the throughputs with idle and busy channel respectively [8].

$$
\begin{aligned}
& R_{0}(\mathrm{~S})=\frac{T-S}{T} C_{0}\left(1-\mathrm{P}_{f}\right) \mathrm{P}\left(\mathrm{H}_{0}\right) \\
& R_{1}(\mathrm{~S})=\frac{T-S}{T} C_{1}\left(1-\mathrm{P}_{d}\right) \mathrm{P}\left(\mathrm{H}_{1}\right)
\end{aligned}
$$

While the achievable throughput for $\mathrm{SU}$ is:

$$
\begin{gathered}
\mathrm{R}_{T}(\mathrm{~S})=R_{0}(\mathrm{~S})+R_{1}(\mathrm{~S}) \\
\mathrm{R}_{T}(\mathrm{~S})=\frac{T-S}{T}\left[C_{0}\left(1-\mathrm{P}_{f}\right) \mathrm{P}\left(\mathrm{H}_{0}\right)+C_{1}\left(1-\mathrm{P}_{d}\right) \mathrm{P}\left(\mathrm{H}_{1}\right)\right]
\end{gathered}
$$

Assuming that the probability $\mathrm{P}(\mathrm{H} 1)$ of $\mathrm{PU}$ is 0.5 and $\mathrm{P}(\mathrm{H} 0)$ is 0.5 such that we do not know the probability at which the channel is idle or busy. Notice that $\mathrm{C}_{0}>\mathrm{C}_{1}$. Thus the first term in (23) controls the achievable throughput.

\section{COOPERATIVE SPECTRUM SENSING}

CR Cooperative spectrum sensing takes place when a group of CRs share the sense information they gain for PU detection. This supply additional accuracy of spectrum sensing over the area where the CRs are located. Cooperative spectrum sensing plays a very significant role in the research of CR due to its capability to enhance sensing performance especially in the fading, shadowing and noise uncertainty [1].Figure 4 shows that the cooperative spectrum sensing has two different channels, 1 from PU to SU called sensing channel and second called reporting channel between SU and the Fusion Canter (FC) at which each SU send the sensing decision to FC and finally the FC take the final decision of existence PU or not 
according to some different rules (AND Rule, OR Rule, and MAJORITY Rule).

\section{A. Logical-OR Rule}

The decision rule of "OR" assigns that if any one of the local decisions sent to the FC is a logical one, the final decision is one (i.e. when at least 1 out of M SUs detect a PU, it is adjudged that a PU signal is present) [10].

$$
\begin{gathered}
\tilde{P}_{d}=1-\prod_{i=1}^{M}\left(1-\mathrm{P}_{d, i}\right) \\
\tilde{P}_{f}=1-\prod_{i=1}^{M}\left(1-\mathrm{P}_{f, i}\right)
\end{gathered}
$$

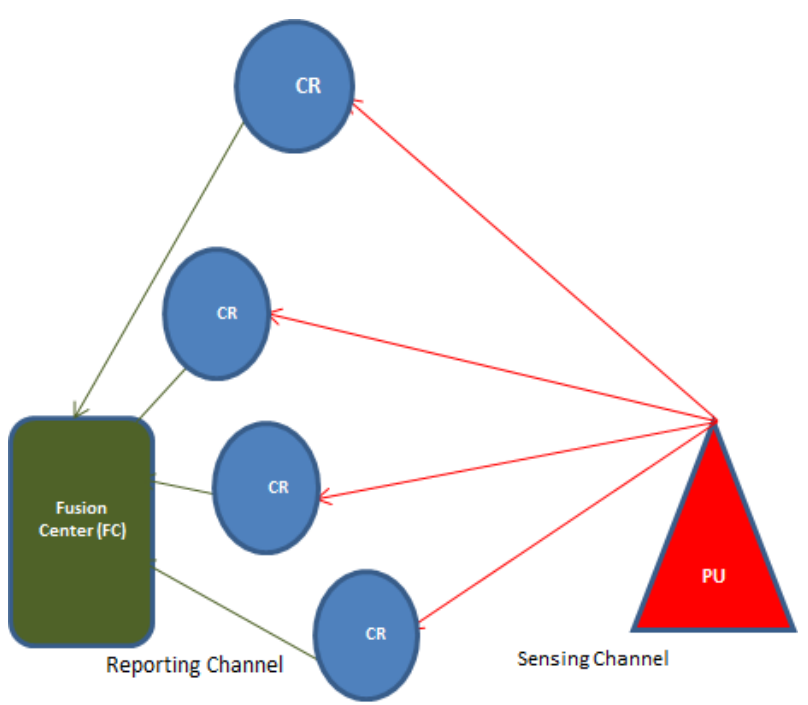

Fig.4. Cooperative Spectrum Sensing Model

\section{B. Logical-AND Rule}

The decision rule of "AND" is used when all the local decisions sent to the FC are one; that makes the final decision being one [10].

$$
\begin{aligned}
\tilde{P}_{d} & =\prod_{i=1}^{M} \mathrm{P}_{d, i} \\
\tilde{P}_{f} & =\prod_{i=1}^{M} \mathrm{P}_{\mathrm{f}, i}
\end{aligned}
$$

\section{Logical MAJORITY Rule}

The decision rule "MAJORITY" is used when half or more of the local decisions sent to the FC are one; that makes the terminal decision to be one.

$$
\begin{aligned}
& \tilde{P}_{f}=\sum_{i=j}^{M}\left(\begin{array}{l}
M \\
i
\end{array}\right) \mathrm{P}_{\mathrm{f}, i}{ }^{i}\left(1-\mathrm{P}_{f, i}{ }^{(\mathrm{M}-i)}\right) \\
& \tilde{P}_{d}=\sum_{i=j}^{M}\left(\begin{array}{l}
M \\
i
\end{array}\right) \mathrm{P}_{\mathrm{d}, i}{ }^{i}\left(1-\mathrm{P}_{\mathrm{d}, i}{ }^{(\mathrm{M}-i)}\right)
\end{aligned}
$$

Where $\mathrm{j}$ is the integer number selected between 1 and $\mathrm{M}$. If $\mathrm{j}$ equals 1 , the fusion rule becomes OR fusion rule. Else if $j$ equals $\mathrm{M}$, the fusion rule becomes AND rule. Throughput will change relying on the type of spectrum sensing if it is single user or cooperative (ED and MTSS). Furthermore, in cooperative spectrum sensing, throughput will change relying on the type of fusion rule. Then the throughput in this case which has two hypotheses is given by [8]:

$$
\tilde{\mathrm{R}}_{T}(\mathrm{~S})=\frac{T-S}{T}\left[C_{0}\left(1-\tilde{\mathrm{P}}_{f}\right) \mathrm{P}\left(\mathrm{H}_{0}\right)+C_{1}\left(1-\tilde{\mathrm{P}}_{d}\right) \mathrm{P}\left(\mathrm{H}_{1}\right)\right]
$$

\section{SIMULATION RESULTS}

The CR frame as shown in Fig.1 the total frame time is $\mathrm{T}=40 \mathrm{~ms}$, sampling frequency $0.1 \mathrm{MHz}$, target probability of detection is set to 0.9 and number of tapers taken is 4. Fig.5 compares between MTM sensing and Conventional sensing (ED) under $\mathrm{SNR}=-12 \mathrm{~dB}$. We can get $\mathrm{Pd}=0.9$ and 0.4 in case of MTM and Conventional Sensing respectively at $\mathrm{Pf}=0.1$ which indicate the enhancement of detection probability in case of MTM more than Conventional sensing.

Fig.6 shows that $\mathrm{Pd}$ of cooperative spectrum sensing is improved compared to conventional sensing ( $\mathrm{SNR}=-15 \mathrm{~dB}$ and $\mathrm{K}=3 \mathrm{CR}$ users) $\mathrm{Pd}=(0.29,0.28,0,3$ and 0.21$)$ for (AND, OR, Majority and Conventional Sensing) respectively in case of $\mathrm{Pf}$ $=0.1$.

Fig.7 shows the enhancement of detection probability $\mathrm{Pd}=(0.9,0.91,0.95$ and 0.3$)$ in case of (AND , OR , Majority and MTSS method) respectively which indicate detection improvement compared to conventional sensing and its cooperative sensing. Cooperative spectrum sensing is used to solve the hidden node problem so we can conclude that MTM sensing can solve the hidden node problem such that $\mathrm{Pd}=0.3$ in case of cooperative spectrum sensing by using energy detection and also MTM sensing $\mathrm{Pd}=0.3$ under same conditions. Also MT cooperative spectrum sensing can be used to enhance the Pd more which will protect the PU signal more and more.

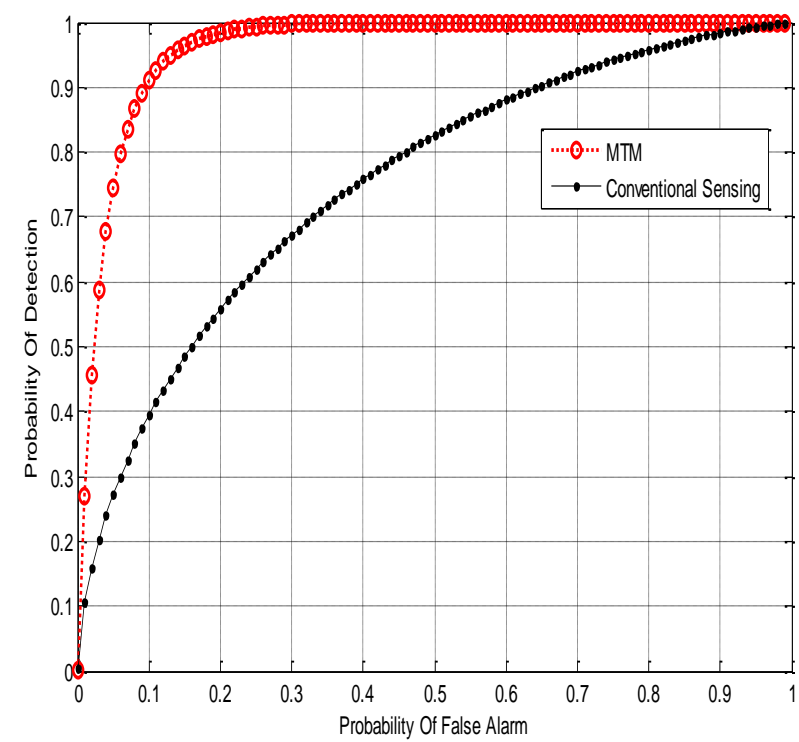

Fig.5. MTM Spectrum Sensing compared to Conventional Sensing $(\mathrm{SNR}=-12 \mathrm{~dB}$ 




Fig.6. Conventional Cooperative Spectrum Sensing compared to Single user Sensing ( $\mathrm{SNR}=-15 \mathrm{~dB}, \mathrm{~K}=3 \mathrm{CR}$ Users)

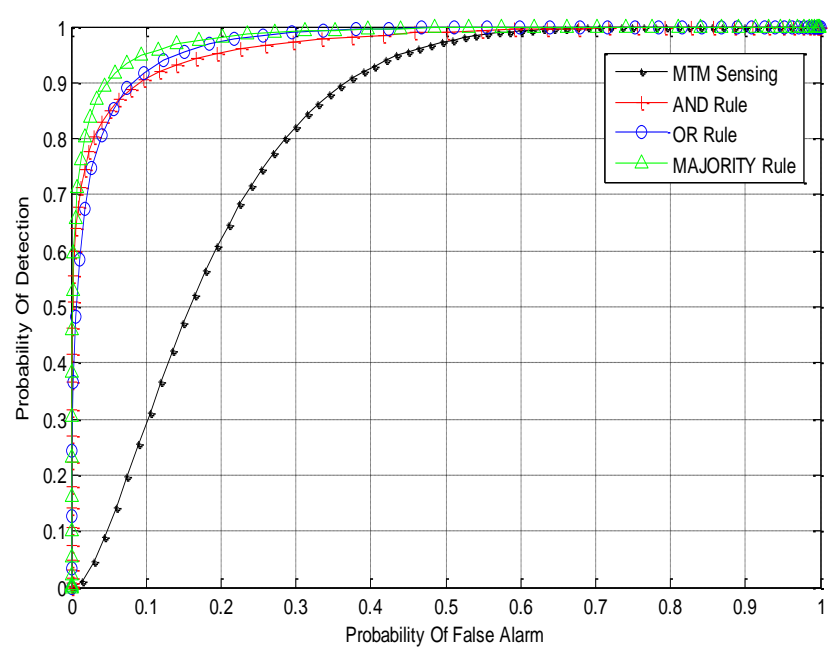

Fig.7. MultiTaper Cooperative Spectrum Sensing compared to Single user Sensing (SNR=-15 dB ,K=3 CR Users)

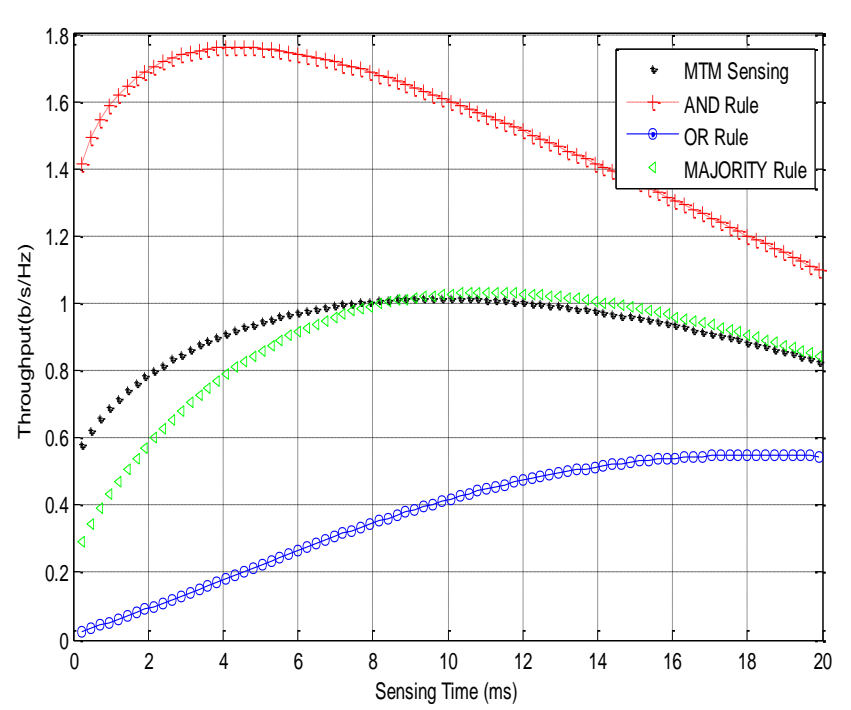

Fig.8. MultiTaper Throughput Vs Sensing time ( $\mathrm{SNR}=-15 \mathrm{~dB}, \mathrm{~K}=3 \mathrm{CR}$ Users)

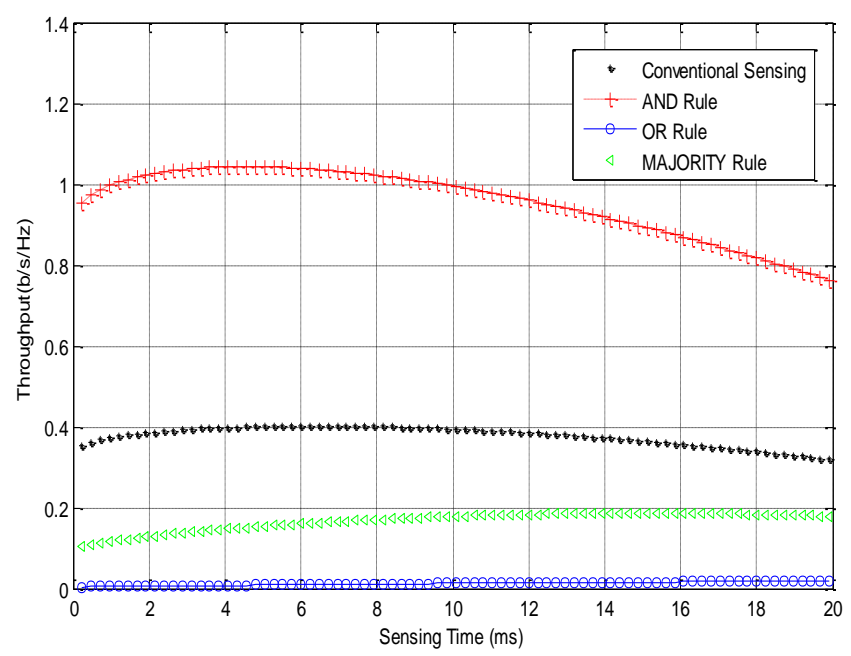

Fig.9. Conventional Throughput Vs Sensing time (SNR=-15 dB ,K=3 CR Users)

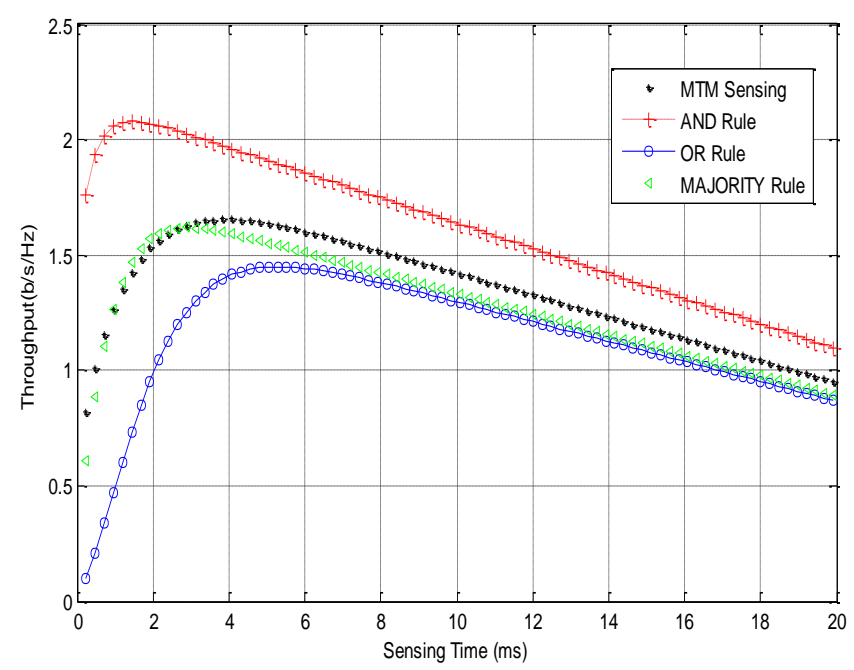

Fig.10. Multi-Taper Throughput Vs Sensing time ( $\mathrm{SNR}=-10 \mathrm{~dB}, \mathrm{~K}=3 \mathrm{CR}$ Users)

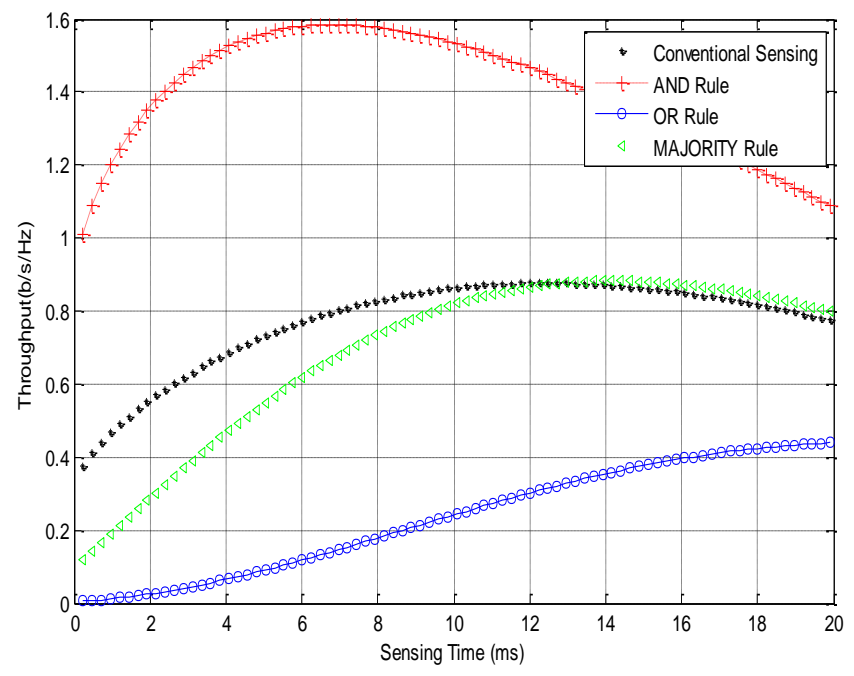

Fig.11. Conventional Throughput Vs Sensing time ( $\mathrm{SNR}=-10 \mathrm{~dB}, \mathrm{~K}=3 \mathrm{CR}$ Users) 
The used conditions ( $\mathrm{SNR}=-15 \mathrm{~dB}, \mathrm{~K}=3$ ) in Fig.8 shows that the optimum achievable throughput for MTM Cooperative Spectrum Sensing is $(1.75,0.55,1,1) \mathrm{b} / \mathrm{s} / \mathrm{Hz}$ in case of (AND , OR , MAJORITY and MTM Sensing) Respectively at sensing time $(4,18,10,10)$ msec respectively.

Comparing results obtained in case of Conventional Cooperative spectrum sensing under same conditions as shown in Fig.9 get Achievable throughput $(1.05,0.001,0.15,0.4)$ $\mathrm{b} / \mathrm{s} / \mathrm{Hz}$ for (AND , OR , MAJORITY, and Conventional Sensing) respectively at sensing time $(4,12,12,4) \mathrm{msec}$.

The throughput in AND rule is the best because the probability of false alarm is minimum as the SU transmits when any user decides that the channel is free. Inversely, the OR rule has a high probability of false alarm because the SU transmits when all SUs decide that the channel is free and the Majority rule has acceptable throughput with high probability of detection. AND rule also optimizes sensing time which has the maximum throughput in low sensing time.

Fig.10 shows the effect of increasing ( $\mathrm{SNR}=-10 \mathrm{~dB}, \mathrm{~K}=3$ CR users) get the achievable throughput $(2.1,1.45,1.65,1.7)$ $\mathrm{b} / \mathrm{s} / \mathrm{Hz}$ at sensing time $(1.5,5,2.8,4) \mathrm{msec}$ in case of (AND, OR ,MAJORITY, MTM Sensing ) respectively but in case of (AND , OR , MAJORITY , Conventional Sensing) the achievable throughput are $(1.6,0.42,0.9,0.9) \mathrm{b} / \mathrm{s} / \mathrm{Hz}$ at sensing time $(7,20,14,12)$ msec respectively as shown in Fig.11.

\section{CONCLUSIONS}

In this paper, a proposed Multi-Taper Spectrum Sensing (MTSS) technique is used to enhance the achievable throughput in a small sensing time. The simulated results indicate that the proposed model is working properly. As the achievable throughput for secondary user in case of proposed MTSS technique has a better values compared to conventional spectrum sensing under different scenarios. MTSS is a good technique for hidden node problem which make more protection for primary user, moreover, MT Co-operative spectrum sensing is used to improve the achievable throughput at low sensing time. Cooperative MT Spectrum sensing is more efficient than Conventional Cooperative spectrum sensing at low SNR of PU. The MTSS AND fusion rule has the maximum throughput but has less detection performance compared to other MT fusion rules. Furthermore study can be done on the future which would study the effect of MT-MIMO (Multi-Taper-Multiple Input Multiple Output) spectrum sensing on sensing achievable throughput in case of different hypothesis including the effect of primary user attack during sensing time.

\section{REFERENCES}

[1] Abdulsattar, Mahmood A., and Zahir A. Hussein. "Energy detection technique for spectrum sensing in cognitive radio: a survey." International Journal of Computer Networks \& Communications 4.5 (2012): 223.

[2] Salam, Ahmed O. Abdul, et al. "Multi-taper and MIMO techniques for spectrum sensing in cognitive radio." Electronics, Circuits, and Systems (ICECS), 2015 IEEE International Conference on. IEEE, 2015.

[3] Taherpour, Abbas, Masoumeh Nasiri-Kenari, and Saeed Gazor. "Multiple antenna spectrum sensing in cognitive radios." IEEE transactions on wireless communications 9.2 (2010): 814-823.

[4] Atapattu, Saman, Chintha Tellambura, and Hai Jiang. "Conventional Energy Detector." Energy Detection for Spectrum Sensing in Cognitive Radio. Springer New York, 2014. 11-26.

[5] Thomson, David J. "Jackknifing multitaper spectrum estimates." IEEE Signal Processing Magazine 24.4 (2007): 20-30.

[6] D. J. Thomson, "Spectrum Estimation and Harmonic Analysis", Proc. IEEE, vol. 70, no. 9, pp. 1055-1096, September 1982.

[7] Owayed, A. Alghamdi, Z. Ahmed Mohammed, and A. Abu-Rgheff Mosa. "Probabilities of detection and false alarm in multitaper based spectrum sensing for cognitive radio systems in AWGN." Communication Systems (ICCS), 2010 IEEE International Conference on. IEEE, 2010.

[8] Yousef, Elsayed M., Heba Y. Soliman, and Atef M. Ghuniem. "SensingThroughput tradeoff with primary user traffic and cooperative sensing in cognitive radio." Computer and Communication Systems (ICCCS), 2017 2nd International Conference on. IEEE, 2017.

[9] Van Drongelen, Wim. Signal processing for neuroscientists: an introduction to the analysis of physiological signals. Academic press, 2006.

[10] Ejaz, Waleed, Ghalib A. Shah, and Hyung Seok Kim. "Energy and throughput efficient cooperative spectrum sensing in cognitive radio sensor networks." Transactions on Emerging Telecommunications Technologies 26.7 (2015): 1019-1030. 